\title{
Relationship between Fork Progression and Initiation of Chromosome Replication in E. coli
}

\author{
Elena C. Guzmán ${ }^{1}$ et al. ${ }^{*}$ \\ ${ }^{1}$ Departamento de Bioquímica, Biología Molecular y Genética, \\ Facultad de Ciencias, Universidad de Extremadura, 06071-Badajoz \\ Spain
}

\section{Introduction}

Ribonucleoside diphosphate reductase (RNR) of Escherichia coli is the prototype of the class I reductases common to most prokaryotes and eukaryotes from viruses to man. It is the only specific enzyme required, under aerobic growth, for the enzymatic formation of deoxyribonucleotides, the precursors for DNA synthesis. DNA replication requires a balanced supply of the four dNTPs, which explains the complex allosteric control of the enzyme (reviewed in Nordlund \& Reichard, 2006). The active enzyme is a 1:1 complex of two subunits called proteins R1 and R2, each consisting of two polypeptide chains, coded by the genes $n r d A$ and $n r d B$, respectively (Hanke \& Fuchs, 1983). Although about 3000 nucleotides have to be consumed per second when a bacterium replicates its chromosome with two replication forks, only a very small pool of dNTP is accumulated in the cells. This pool would permit replication for no longer than half a minute (Werner, 1971; Pato, 1979). Channeling of the biosynthesis and compartmentation of the precursors has been proposed as explanations of how this shortage may be circumvented (Mathews, 1993; Kim et al., 2005) To satisfy the changing demand for the four deoxynucleotides, RNR must be closely associated with the replication machinery. In the aforementioned studies, Mathews et al., found evidence for the association of this enzyme with others related to the precursor biosynthesis, and coined the term dNTP-synthesizing complex (Mathews, 1993).

The best-known defective RNR mutant in E. coli contains a thermolabile R1 subunit, coded by the $\operatorname{rdA101}$ allele. This allele carries a missense mutation, causing a change in amino acid 89 (L89P) (Odsbu et al., 2009). This leucine-to-proline substitution is close to the ATP cone domain that is located in the $\mathrm{N}$-terminal region of the $\mathrm{R} 1$ protein and is, according to the holoenzyme model, located close to the R1-R2 interaction surface (Uhlin \& Eklund, 1994), although no structural analysis of the mutant protein has been performed.

\footnotetext{
* Israel Salguero², Carmen Mata Martín'1, Elena López Acedo'1, Estrella Guarino², $\mathrm{M}^{\mathrm{a}}$ Antonia Sánchez-Romero ${ }^{3}$, Vic Norris ${ }^{4}$ and Alfonso Jiménez-Sánchez

${ }^{2}$ Department of Zoology, University of Oxford, South Parks Road, Oxford OX1 3PS, United Kingdom.

${ }^{3}$ School of Biosciences. University of Birmingham. Edgbaston. Birmingham B15 2TTUK.

${ }^{4}$ Laboratoire d'Assemblages moléculaires: modélisation et imagerie SIMS, EA 3829, Faculté des Sciences de l'Université de Rouen, 76821 Mont Saint Aignan Cedex, France.
} 
The RNR101 protein is inactivated at $42^{\circ} \mathrm{C}$ in vitro after 2 min (Fuchs et al., 1972), although a thermoresistant period of $50 \mathrm{~min}$ has been observed in vivo sustaining a relative increase in the amount of DNA up to $45-50 \%$ in the nrdA101 mutant strain (Guzmán et al., 2002). Furthermore, it has been shown by flow cytometry that the $n r d A 101$ mutant is able to replicate the entire chromosome in the presence of rifampicin at $42^{\circ} \mathrm{C}$ (Guzmán et al., 2002; Fig. 1). The pool of free dNTPs is not responsible for this DNA synthesis, as inhibition of RNR activity by hydroxyurea caused an immediate cessation of dNTP incorporation either in the presence or in absence of rifampicin (Fig. 1). Marker frequency analysis and flow cytometry show that this chain elongation of DNA replication in the nrdA101 mutant does not end at the terminus of replication but stops stochastically throughout the chromosome (Guzmán et al., 2002).

These results are consistent with RNR having a thermoresistance period due to protection by some subcellular structure. This enzyme has been proposed to be part of a complex for the biosynthesis of dNTP (Mathews, 1993) therefore the association with this complex might explain such protection. We have proposed that, as a component of the replication hyperstructure, the RNR101 protein would be protected from thermal inactivation and that this would suffice to allow chromosome replication for $50 \mathrm{~min}$ in restrictive conditions (Guzmán et al., 2002; 2003, Molina \& Skarstad, 2004; Guarino et al., 2007a; 2007b; Riola et al., 2007).

Supporting this model, RNR has been colocalized with the replisome-associated proteins DnaB helicase and DNA polymerase $\tau$ subunit, and with the fork-associated protein SeqA (Fig. 2) (Sánchez-Romero et al., 2010).
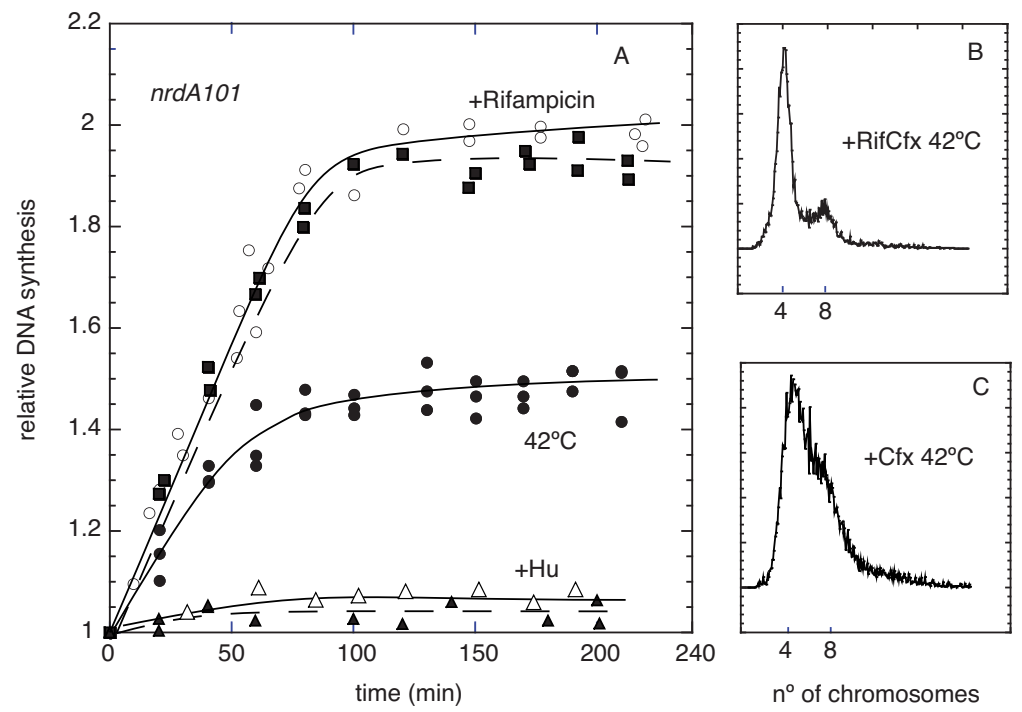

Fig. 1. (A) Runout DNA synthesis of the $n r d A 101$ strain in the presence of rifampicin (open circles) or in the presence of rifampicin and hydroxyurea at $30^{\circ} \mathrm{C}$ (open triangles), or after a shift from $30^{\circ} \mathrm{C}$ to $42^{\circ} \mathrm{C}$ in the absence (closed circles) or in the presence of rifampicin (closed squares) or in the presence of rifampicin and hydroxyurea (closed triangles). Flow cytometry profile after $4 \mathrm{~h}$ in cephalexin at $42^{\circ} \mathrm{C}$ with (B) or without (C) rifampicin. Data were adapted from Guzmán et al. 2002. 


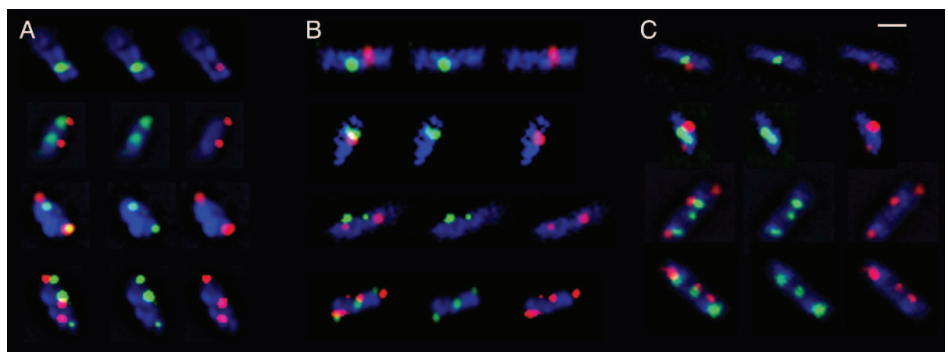

Fig. 2. Fluorescence microscopy of tagged-NrdB and SeqA or DnaB or DnaX. Fluorescence microscopy images of (A) CMT935 (nrdB::3×FLAG dnaB::HA) immunolabeled with Cy3anti-FLAG (red) and FITC-anti-HA (green), (B) CMT936 (nrdB::3×FLAG dnaX::HA) immunolabeled with the same antibodies, (C) CMT931 (nrdB::3×FLAG) immunolabeled with Cy3-conjugated anti-FLAG (red) and FITC-anti-SeqA (green) antibodies (C). Cells were also stained with Hoechst 33258 for nucleoid visualization. Each group of cells shows nucleoid (blue) and, from left to right: both green and red, only green, and only red fluorescence. The bar represents $1 \mu \mathrm{m}$. Data were adapted from Sánchez-Romero et al., 2010.

Furthermore, a hyperstructure containing RNR101 impairs replication fork progression even at the permissive temperature (Guarino et al., 2007a). Arrest of replication forks is known to cause double-strand breaks, DSBs (Bierne \& Michel, 1994; Kuzminov, 1995). We have shown that the number of DSBs in the $n r d A 101 \mathrm{recB}$ strain is greater than that in the strain $n r d A+$ $r e c B$, consistent with an increase in the number of the stalled forks due to the presence of the deficient replication fork reversal (RFR) even at the permissive temperature. These DSBs are generated by RuvABC, a specific resolvase for Holliday junctions. According to the RFR model (Michel et al., 2004), these results indicate the occurrence of replication fork reversal as the mechanism for restarting stalled replication forks. These results indicate that the lengthening of the $C$ period in the $n r d A 101$ mutant strain growing at $30^{\circ} \mathrm{C}$ results not only from the reduced activity of RNR101 but also from the impaired progression of replication forks. The Tus protein is known to arrest replication forks through specific interaction with ter sequences by antagonizing the activity of the replicative helicase (reviewed by Bussiere \& Bastia, 1999). Hence, the progression of the replication forks might be improved by the absence of this protein. In support of this idea we have found a decrease in the number of stalled replication forks in a $n r d A 101$ recB Dtus triple mutant strain (Table 1).

\begin{tabular}{lll}
\hline Relevant phenotype & \multicolumn{2}{l}{ \% linear DNA a } \\
\hline$n$ rdA+ & 4.58 & \pm 2.51 \\
$n r d A+\operatorname{rec} B$ & 15.18 & \pm 2.83 \\
$n r d A+\operatorname{rec} B$ ruv $A B C$ & 6.74 & \pm 2.60 \\
$n r d A 101$ & 5.72 & \pm 1.41 \\
$n r d A 101$ recB & 24.79 & \pm 7.05 \\
$n$ rdA101 recB Dtus & 12.64 & \pm 4.70 \\
$n r d A 101$ recB ruvABC & 5.94 & \pm 2.19 \\
\hline
\end{tabular}

Table 1. Growing the $n r d A 101 \mathrm{recB}$ mutant strain at $30^{\circ} \mathrm{C}$ increases RuvABC-dependent

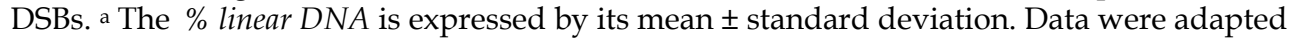
from Guarino et al., 2007a. 
It is intriguing that rifampicin or cloramphenicol addition, as well as the presence of a dnaAts allele, allowed the completion of chromosome replication in the nrdA101 mutant at the high temperature (Guzmán et al., 2002, Salguero et al., 2011). Inhibition of RNA and of protein synthesis, and inactivation of the DnaA protein all inhibit initiation of chromosome replication; therefore, completion of chromosome replication in the $n r d A 101$ strain at $42^{\circ} \mathrm{C}$ could be ascribed to the inhibition of new DNA initiations (Salguero et al., 2011). We suggest that the replication of the entire chromosome that occurs at the non-permissive temperature when new initiations are inhibited is due to a more efficient elongation as a consequence of the decreasing number of forks per chromosome.

In studying replication in the $n r d A 101$ mutant, we used several conditions to reduce the overlap of replication rounds ( $n$, Sueoka \& Yoshikawa, 1965) and, consequently, the number of replication forks per chromosome $\left(2^{n+1}-2\right)$. We found an inverse correlation between this overlap and the amount of DNA that can be synthesized by the $n r d A 101$ strain at the restrictive temperature.

Consequently, we propose that a reduction in the number of forks replicating the chromosome results in an improvement in the quality of replication that allows the deficient replication hyperstructure of the nrdA101 strain to be more processive at the high temperature. This proposal points toward the co-regulation of the elongation rate and the initiation frequency as a general control mechanism in prokaryotic and eukaryotic replication.

\section{Reduction in the overlap of replication rounds improves fork progression at the restrictive temperature in a nrdA101 strain}

We have previously shown that, due to an elongation of the replication period lasting more than twice the cell cycle at $30^{\circ} \mathrm{C}(C=186 \mathrm{~min}, \tau=79 \mathrm{~min})$, the $n r d A 101$ strain undergoes multifork replication resulting in two thirds of cells containing one or two chromosomes with 6 forks per chromosome, and one third of cells with a chromosome containing 14 forks (Guzmán et al., 2002). After a temperature shift from $30^{\circ} \mathrm{C}$ to $42^{\circ} \mathrm{C}$, these cells replicate their DNA for $50 \mathrm{~min}$, giving a runout synthesis of 52 per cent (Table 2). To test whether a reduction in the number of replication forks at $30^{\circ} \mathrm{C}$ could improve the ability of forks to replicate the chromosome at $42^{\circ} \mathrm{C}$ in the nrdA101 strain, we reduced that number by different methods.

\subsection{Experimental approach}

In contrast with eukaryotic organisms, the time required to replicate a single chromosome (C) in E. coli can be longer than the generation time $(\tau)$, and in these conditions an overlap of replication rounds is obtained. The degree of overlap can be quantified by $n$, defined as the $C$ to $\tau$ ratio (Sueoka \& Yoshikawa, 1965; Cooper \& Helmstetter, 1968). $n$ was determined by using the $\Delta G$ value, which is defined as the relative runout DNA synthesized after inhibiting new initiation events, while ongoing forks are allowed to finish (Pritchard \& Zaritsky, 1970). This experimental condition can be achieved by the addition of rifampicin $(150 \mu \mathrm{g} / \mathrm{ml})$, which inhibits RNA polymerase whose activity is known to be required for the initiation step. From the experimental $\Delta G$ value, $n$ can be calculated from the algorithm $\Delta \mathrm{G}=\left[2^{n} n \ln 2 /\left(2^{n}-1\right)\right]-1$ (Sueoka \& Yoshikawa, 1965). Thus, the relative amount of the DNA synthesized after inhibition of initiation of replication only depends on the number of replication cycles per chromosome before the inhibition. The $\Delta \mathrm{G}$ values under any growth 
condition were obtained according to Pritchard and Zaritsky (Pritchard \& Zaritsky, 1970; Zaritsky \& Pritchard, 1971), and $n$ was obtained by the use of a computer software developed in our lab (Jiménez-Sánchez \& Guzmán, 1988). As the $\Delta \mathrm{G}$ algorithm requires the completion of chromosome replications under any treatment, additional flow cytometry analysis is necessary to verify this completion. Flow cytometry analysis was performed in the presence of rifampicin $(150 \mu \mathrm{g} / \mathrm{ml})$ and cephalexin $(50 \mu \mathrm{g} / \mathrm{ml})$ to inhibit cell division. Thus at the end of the treatment with the drugs, cells should contain $2^{\text {(integer n) }}$ or $2^{\text {(integer } n+1)}$ fully replicated chromosomes if they display synchronous initiation (Skarstad et al., 1985).

As explained above, the overlap of the replication rounds depends on two parameters, the generation time, $\tau$, and the $C$ period. In this study we used conditions that affect both parameters. The generation time was altered by growing the cells in glycerol or arabinose media. The $C$ period was reduced by several methods such as by the presence of the dnaA174 allele, by increasing the number of copies of datA sites in a plasmid, or by deletion of the DARS2 reactivating sequence. To determine the effect of several replication overlaps on DNA synthesis in the $n r d A 101$ strain at $42^{\circ} \mathrm{C}$, we compared the residual DNA synthesis at $42^{\circ} \mathrm{C}$ relative to the runout after rifampicin at $30^{\circ} \mathrm{C}$ (i.e. $42^{\circ} \mathrm{C} / \Delta G$ at $30^{\circ} \mathrm{C}$ ) with the $n$ value (the $C$ to $\tau$ ratio).

\subsection{Increasing the generation time}

We lowered $n$ by increasing the generation time using different carbon sources, such as glucose, arabinose or glycerol. Cultures of the $n r d A 101$ strain were grown at $30^{\circ} \mathrm{C}$, in MM9 media containing one of the carbon sources, in the presence of ${ }^{3} \mathrm{H}$-thymidine to label the newly synthesized DNA. When cultures reached mid-exponential phase $\left(0.1 \mathrm{OD}_{550}\right)$, two samples were taken, one to be incubated at $42^{\circ} \mathrm{C}$, the non-permissive temperature, and the second one to be treated with rifampicin $(150 \mu \mathrm{g} / \mathrm{ml})$ at $30^{\circ} \mathrm{C}$ to inhibit new initiations of chromosome replication. DNA synthesis was measured for $4 \mathrm{~h}$ (as the acid-precipitable radioactive material) and the values relative to the radioactive material incorporated at the beginning of treatment were represented. The values obtained in several strains and growth media are given in Table 2. To verify the completion of replication rounds, flow cytometry analysis was performed in the presence of rifampicin $(150 \mu \mathrm{g} / \mathrm{ml})$ and cephalexin $(50 \mu \mathrm{g} / \mathrm{ml})$, which inhibits cell division (data not shown).

As expected from growing the bacteria in a carbon source different from glucose, a lengthening of the generation time and a lowering in the number of overlapped replication rounds, $n$, were observed (Table 2). After the shift to $42^{\circ} \mathrm{C}$ in these media, the amount of synthesized DNA was inversely correlated with the number of previous overlaps. These results suggest that a reduction in the overlap of the replication rounds increases the capability to synthesize DNA for a longer period of time after the shift to $42^{\circ} \mathrm{C}$.

\subsection{Reducing the $C$ period}

\subsubsection{By the presence of $d n a A$ defective alleles}

The presence of $d n a A$ defective alleles has been reported to reduce the time required for chromosomal elongation (C period) at permissive conditions and this effect might suppress the defects in replication of some DNA elongation mutants (Torheim et al., 2001; Skovgaard \& Lobner-Olesen, 2005). We used the dnaA174 allele, which codes for a non-thermosensitive DnaA protein with a high ATPase activity, which in turn maintains a low DnaA-ATP level associated with a decreased the $C$ period (Gon et al., 2006). We found the nrdA101 dnaA174 double mutant had an overlap of replication cycles at $30^{\circ} \mathrm{C}$ that decreased from 2.36 to 1.37 
and a five fold increased capability to synthesize DNA at $42^{\circ} \mathrm{C}$ relative to the $n r d A 101$ single mutant (Fig. 3, Table 2).

When incubated for $4 \mathrm{~h}$ at $42^{\circ} \mathrm{C}$ in the presence of cephalexin, the DNA content per cell in nrdA101 dnaA174 double mutant is higher than observed under completion of the ongoing chromosome replication rounds; although the flow cytometry profile showed a broad distribution with no discrete peaks corresponding to completed chromosomes, as observed in the $n$ rdA101 strain (Fig. 3).

We have verified that the number of overlapped replication forks per chromosome at $30^{\circ} \mathrm{C}$ could be also lowered by introducing dnaA46, dnaA5 or dnaA508 alleles in the nrdA101 background (Table 2; Salguero et al., 2011). After incubation at $42^{\circ} \mathrm{C}$, all nrdA101 dnaAts strains exhibited a relative DNA synthesis and a thermoresistant period similar to those obtained by rifampicin addition either at $30^{\circ} \mathrm{C}$ or $42^{\circ} \mathrm{C}$. As DnaA protein is required for chromosomal initiation and as all these alleles code for a thermosensitive DnaA protein, the runout value at the restrictive condition is the highest value that can be expected.

These results suggest that a lowering in the number of replication forks running along the chromosome could improve the progression of replication in the $n r d A 101$ mutant at the restrictive condition. DnaA is also known to have a regulatory control in the transcription of several genes, including $d n a A, m i o C, r p o H$ and the $n r d A B$ operon (reviewed in Messer \& Weigel, 2003; Gon et al., 2006; Herrick \& Sclavi, 2007; Olliver et. al., 2010); moreover, the $n r d A$ gene is over-expressed in the presence of a defective DnaA protein (Gon et al., 2006, Lobner-Olesen et al., 2008). This over-expression is, however, unlikely to be responsible for the high residual DNA synthesis found in the $n r d A 101$ strain at $42^{\circ} \mathrm{C}$ since the chromosome is fully replicated at $42^{\circ} \mathrm{C}$ in all studied $n r d A 101$ dnaA strains even in the absence of protein synthesis (and hence absence of overproduced NrdA) (Salguero et al., 2011).

In addition, over-expression of the $n r d A B$ operon from a plasmid, leading to a doubling of enzyme activity as measured in cell-free extracts, causes only a doubling of the dATP, dCTP and dTTP pools without any increase in the dGTP pool (Wheeler et al., 2005). Consequently, an increase in the replication rate should not be expected as dGTP pool would be limiting. Moreover, it is far from certain that an overproduction of RNR outside the replication

\begin{tabular}{llllllll}
\hline Strains & $\tau$ & $\Delta \mathrm{G} 30^{\circ} \mathrm{C}$ & $42^{\circ} \mathrm{C}$ & $42^{\circ} \mathrm{C} / \Delta \mathrm{G} 30^{\circ} \mathrm{C}$ & $\mathrm{n}$ & ori/ter & $\mathrm{C}$ \\
\hline nrdA+ & 78 & 0.55 & $\mathrm{nt}$ & $\mathrm{nt}$ & 1.37 & 2.58 & 107 \\
nrdA101 & 79 & 1.03 & 0.52 & 0.50 & 2.36 & 5.13 & 186 \\
nrdA101 arabinose & 105 & 0.59 & 0.83 & 1.40 & 1.46 & 2.75 & 153 \\
nrdA101 glycerol & 112 & 0.35 & 0.58 & 1.65 & 0.91 & 1.87 & 102 \\
nrdA101 dnaA174 & 78 & 0.55 & 1.40 & 2.54 & 1.37 & 2.58 & 107 \\
nrdA101 dnaA46 & 80 & 0.45 & 0.48 & 1.06 & 1.15 & 2.21 & 92 \\
nrdA101 dnaC2 & 82 & 1.00 & 0.45 & 0.45 & 2.30 & 4.92 & 188 \\
nrdA101/pMOR6 & 81 & 0.73 & 0.70 & 0.95 & 1.76 & 3.38 & 142 \\
nrdA101 DARS2 & 85 & 0.55 & 0.95 & 1.80 & 1.26 & 2.39 & 107 \\
\hline
\end{tabular}

Table 2. Cell cycle parameters from the nrdA101 strain growing in MM9 with different carbon sources or containing a second mutant allele. All strain were grown in MM9 medium with glucose except otherwise indicated. $\Delta \mathrm{G} 30^{\circ} \mathrm{C}$, relative increase of the amount of DNA after rifampicin addition at $30^{\circ} \mathrm{C} .42^{\circ} \mathrm{C}$, relative increase of the amount of DNA after a shift to $42^{\circ} \mathrm{C}$. $n$, number of replication rounds per chromosome or overlapping degree. ori $/$ ter $=2^{n}$ or number of origins per chromosome. $C$ elongation time $(\min )$ from $C=n \tau$. 

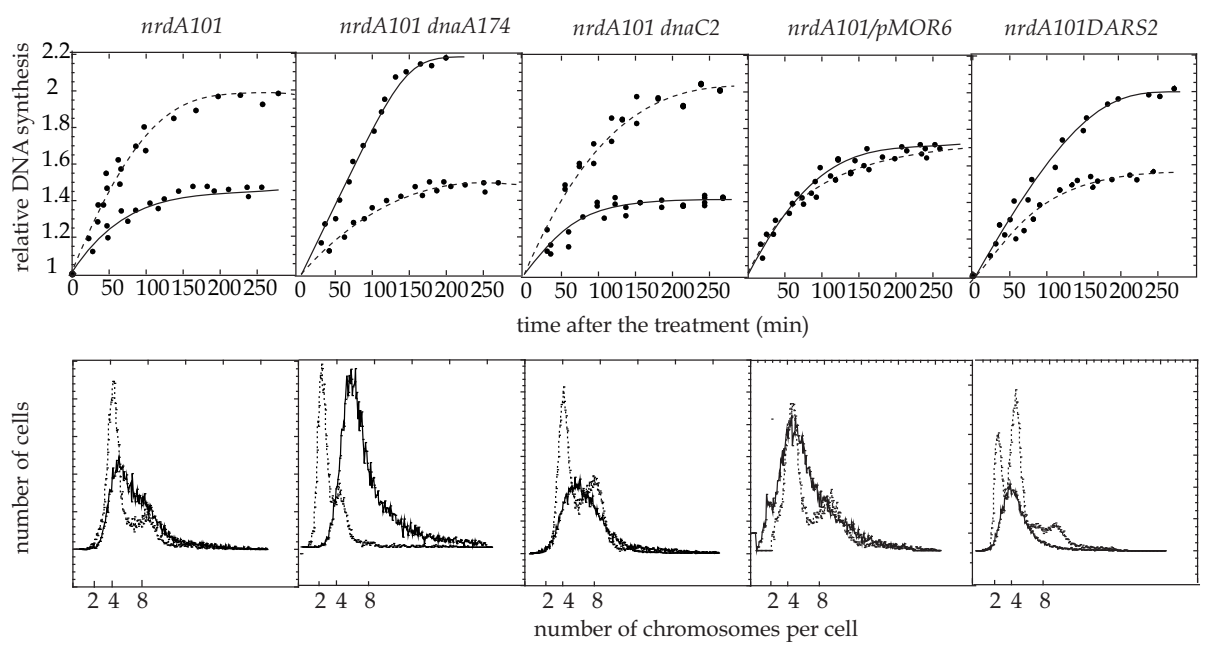

Fig. 3. DNA synthesis (upper panels) after rifampicin addition (dashed line) or incubation at $42^{\circ} \mathrm{C}$ (solid line), and flow cytometry profiles (lower panels) after 4 hours of incubation at $30^{\circ} \mathrm{C}$ in the presence of rifampicin and cephalexin (dashed line) or after 4 hours at $42^{\circ} \mathrm{C}$ in the presence of cephalexin (solid line) in strains nrdA101, nrdA101 dnaA174, nrdA101 dnaC2, $n r d A 101 / p M O R$ and $n r d A 101$ DARS2

hyperstructure would increase the supply of dNTP to the replication enzymes (Pato, 1979; Mathews, 1993).

It has been shown that the $n r d A B$ operon is also over-expressed in a $d n a C 2$ mutant and that, when incubated at the high temperature, 18 per cent of the cells failed to complete chromosome replication (Lobner-Olesen et al., 2008). This observation has been explained by the implication of the DnaC protein in the restart of stalled replication forks during elongation (Maisnier-Patin et al., 2001). We measured DNA synthesis at $30^{\circ} \mathrm{C}$ with rifampicin and at $42^{\circ} \mathrm{C}$ in the $n r d A 101 d n a C 2$ strain; this strain had about the same $\tau$ and $C$, hence $n$, at $30^{\circ} \mathrm{C}$ as the parental $n r d A 101$ strain (Table 2). As expected from these cell cycle parameters, the amount of DNA synthesized at $42^{\circ} \mathrm{C}$ was also similar (Fig. 3). Consequently, over-expression of the $n r d A B$ operon cannot explain the extensive thermoresistant replication found in the $n r d A 101$ strain when new initiations are prevented.

\subsubsection{By increasing the number of copies of the dat $A$ sequence}

The E. coli genome contains 308 DnaA boxes (TTAT(C/A)CA(C/A)A) with variable affinity to DnaA (Schaper \& Messer 1995). A strong DnaA-binding region, datA (from DnaA titration) containing five boxes, has been identified among them (Kitagawa et al., 1996). The datA site titrates unusually large amounts of DnaA protein in vivo (Kitagawa et al., 1996) and it has been suggested that the datA-bound DnaA molecules act as a reservoir of DnaA (Kitagawa et al., 1998). Recently, it has been found that high levels of $\operatorname{dat} A$ completely shut down initiation of replication, whilst moderate levels of datA increase the replication rate relative to that of the wild type (Morigen et al., 2001; 2003). Using this feature we constructed an nrdA101 strain harboring the pMOR6 plasmid, a derivative of the moderate copy number plasmid pACYC177 (Morigen et al., 2001). We found a shortened $C$ period and a lower overlap of replication cycles (Table 2, Fig. 3). Consistent with this, the 
$n r d A 101 / p M O R 6$ strain synthesizes more DNA at the restrictive temperature than its $n r d A 101$ parental strain (Fig. 3). After $4 \mathrm{~h}$ of incubation at $42^{\circ} \mathrm{C}$ we found similar amount of DNA synthesis, either in the presence or in the absence of rifampicin at restrictive conditions. This result differs from that obtained in the $n r d A 101$ mutant, whose DNA synthesis at $42^{\circ} \mathrm{C}$ is half of the observed at $42{ }^{\circ} \mathrm{C}$ when new initiations were inhibited (Table 2) (Salguero et al., 2011). As DNA initiation is not inhibited in the $n r d A 101 / p M O R 6$ strain after the shift to $42^{\circ} \mathrm{C}$, none fully replicated chromosomes were detected at $42^{\circ} \mathrm{C}$ (Fig. 3).

\subsubsection{By deleting the DARS sequence}

The DnaA protein is a member of the AAA+ ATPase family and has an exceptionally high affinity for ATP/ADP (Sekimuzu et al., 1987; Kaguni, 2006). The level of cellular ATP-DnaA oscillates during the replication cycle, peaking around the time of initiation (Kurokawa et al., 1999).

Katayama's group has recently found two chromosomal intergenic regions termed DARS1 and DARS2 (DnaA-reactivating sequence) that directly promote regeneration of ATP-DnaA from ADP-DnaA by nucleotide exchange resulting in the promotion of replication initiation in vitro and in vivo. Deletion of DARS results in decrease in the ATP-DnaA level, causing synthetic lethality with dnaAts and suppression of over-initiation in defective seqA, datA and hda mutants (Fujimitsu et al., 2009). These effects led us to infer that, in the absence of DARS sequences, the nrdA101 DARS mutant would decrease DnaA effective protein and consequently a reduction of the $C$ period would be expected. We found the expected decrease in the $C$ period and in the overlap of replication cycles at $30^{\mathrm{a}} \mathrm{C}$, with a reduction in the number of chromosomes per cell (Table 2, Fig. 3). After 4 hours of incubation at $42^{\circ} \mathrm{C}$, the flow cytometry profile showed a broad distribution of the DNA content per cell, although the capability to synthesize DNA at the restrictive temperature increased three times relative to the single mutant $n r d A 101$ (Table 2, Fig. 3).

Our data show that decreasing the number of replication rounds (ori/ter ratio) correlates with an improved capacity to synthesize DNA in the nrdA101 mutant at the restrictive temperature (Fig. 4). Given that the progression of the replisome is affected in this mutant (Guarino et al., 2007a), we propose there is an improvement in the progression of the replication forks at $42^{\circ} \mathrm{C}$ as a consequence of lowering the number of the replication rounds along the chromosome (ori/ter ratio). According to the model where the RNR is a component of the replication hyperstructure (Guzmán et al., 2002), it is reasonable to think that the defective fork progression observed in this mutant can be alleviated by reducing the number of replication forks running along the chromosome. Consistent with this, the presence of $d n a A$ defective alleles, dnaA(Sx), suppresses the detrimental effect on DNA replication observed in mutants that have problems with the progression of forks due to the presence of defective subunits of DNA polymerase III coded by the dnaX gene (Gines-Candelaria et al., 1995; Blinkova et al., 2003; Skovgaard \& Lobner-Olesen 2005).

Furthermore, a lower availability of wild type DnaA protein induced by the presence of extra copies of the datA sequence alleviates replication problems in both the dnaX (Skovgaard \& Lobner-Olesen, 2005) and the nrdA101 mutant (this work), whilst initiation defects caused by deletion of DnaA box R4 suppress replication elongation defects (Stepankiw et al., 2009).

These observations, together with our data, are consistent with the idea that the progression of replication forks is not merely responsive to elongation factors (dNTP pools or proteins engaged in elongation) but also to the number of forks running along the chromosome. We 
suggest that the best explanation for the reduction of the $C$ period in the results discussed above is a reduction in the number of forks per chromosome or a decrease in the extent of overlapping of replication rounds. Thus, under conditions where ori/ter is high the cells could experience at least two changes. One could be a possible scarcity of hyperstructure components such that increasing the number of hyperstructures increases the probability that they are incomplete and relatively ineffective; hence, reciprocally, restricting the number of replication hyperstructures would increase the probability they contain all the components needed for fully effective replication. In this sense, the suppression of $d n a E$ mutation by the deficiency of enzymes engaged in the glycolysis in Bacilus subtilis, has been explained by a differential composition of the replication hyperstructures that would affect the replication rate (Jannière et al., 2007). Another change could be in the structural constraints caused by the proximity of the replication forks belonging to overlapped replication rounds (Odsbu et al., 2009). In agreement with our explanation, Zaritsky A. et al. have proposed the existence of an 'eclipse' in terms of a minimal distance ( $l \mathrm{~min})$ that the replication forks must move away from oriC before oriCs can 'fire' again (Zaritsky et al., 2007). Our explanation can readily accommodate the proposal of an obligatory, minimal distance between replication forks. The greater the number of the replication rounds per chromosome, the shorter the distance between the replication forks. Thus, the distance between the replication forks could explain the differential progression of the forks along the chromosome in the strains discussed here.

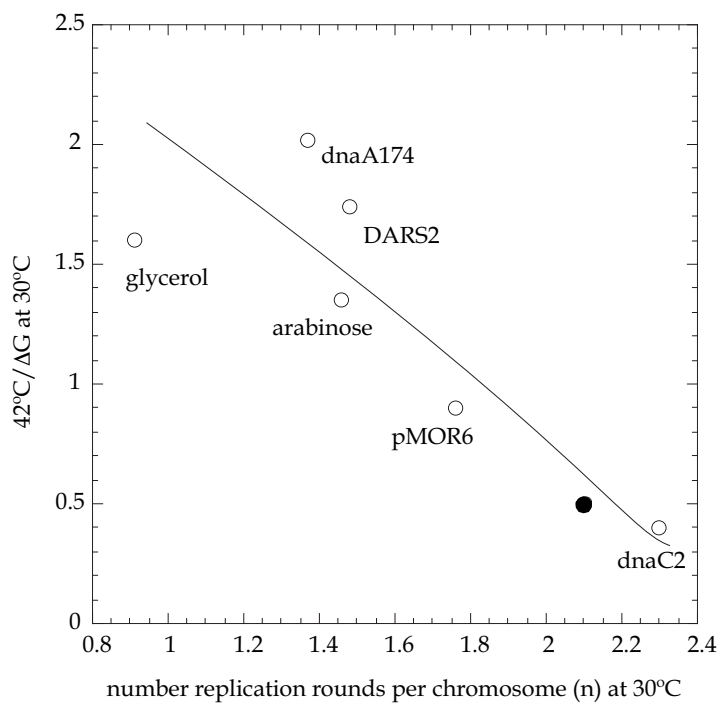

Fig. 4. Relationship between the residual DNA synthesis at $42^{\circ} \mathrm{C}$ relative to the runout after rifampicin at $30^{\circ} \mathrm{C}$ (i.e. $42^{\circ} \mathrm{C} / \Delta G$ at $30^{\circ} \mathrm{C}$ and the $n$ value (the $C$ to $\tau$ ratio) in $n r d A 101$ strain growing in the indicated media or containing the depicted alleles growing in glucose MM9 medium. The black point shows the value of this relationship in the nrdA101 strain growing in glucose MM9 medium. 


\section{Stalled multifork chromosomes as the cause of aberrant DNA segregation and cell death in the nrdA101 mutant at the restrictive temperature}

Growth of $n r d A 101$ strain at the restrictive temperature causes aberrant nucleoid segregation (Guzmán et al., 2003; Riola et al., 2007; Odsbu et al., 2009). This aberrant nucleoid segregation leads to breakdown of the coupling between replication and cell division (Dix \& Helmstetter, 1973; Riola et al., 2007) causing filamentation and cell death. These problems could be related to the fact that DNA replication stops stochastically in the $n$ rdA101 strain at $42^{\circ} \mathrm{C}$ to generate stalled replication forks along the multiforked chromosome (Fig. 3). Similar problems have been observed under other conditions, including UV irradiation, thymine starvation, and mitomycin treatments, inversion of the Ter sequences (Jaffe et al., 1986; Hill et al., 1997), and in dnaN59ts and dnaG2903ts mutants, where the problems have also been attributed to stalled replication forks (Kawakami et al., 2001; Grompe et al., 1991).

Cell viability was studied in all the growth media and strains described above. Cells were grown at $30^{\circ} \mathrm{C}$ and when the cultures reached mid-logarithmic phase (about $0.1 \mathrm{OD}_{550}$ ), an aliquot of each culture was incubated at $42^{\circ} \mathrm{C}$ and the number of viable cells were estimated by serial dilution and plating on rich medium at $30^{\circ} \mathrm{C}$. Viability is expressed relative to the onset of treatment. Growing nrdA101 cells in different carbon sources resulted in different values of cell cycle parameters with a higher number of replication overlaps in glucose than in glycerol medium (Table 2 ) and a greater lethality after the incubation at $42^{\circ} \mathrm{C}$ (Fig. 5). Loss of viability of the $n r d A 101$ strain at the high temperature was completely suppressed by the presence of $d n a A 174$ allele, by extra copies of $d a t A$, or by deleting DARS2 sequence from the chromosome (Fig. 6). The ensemble of these results (Table 2, Fig. 5, Fig. 6) reveals a direct correlation between lethality at high temperature and replication overlapping. This correlation might be explained by either the higher number of sensitive targets (i. e. the replication forks) at $42^{\circ} \mathrm{C}$, the greater vulnerability of sensitive targets due to more replication overlaps, or by an increase in the number of defective replication hyperstructures. These explanations are not mutually exclusive and we consider all as equally likely.

Nucleoid segregation analysis was performed in aliquots of the cultures incubated $42^{\circ} \mathrm{C}$ in the presence of cephalexin $(50 \mu \mathrm{g} / \mathrm{ml})$ for 4 hours plus, during the last $20 \mathrm{~min}$, chloramphenicol addition $(200 \mu \mathrm{g} / \mathrm{ml})$ to condense nucleoids. Micrographs of DAPI stained cells show a high number of cells containing an abnormal number of nucleoids randomly distributed along the filaments (Fig. 5) (Riola et al., 2007). An increased number of cells containing normal and well-segregated nucleoids were found in cells grown in arabinose or in glycerol (Fig. 5). The anomalous number and distribution of nucleoids found in the nrdA101 strain grown at $42^{\circ} \mathrm{C}$ were almost fully suppressed by the presence of dnaA174 allele, by the presence of plasmid pMOR6, which increases the datA sequence copy number, or by the absence of DARS sequences (Fig. 6).

The above results reveal a good correlation between the overlap of replication rounds and aberrant nucleoid segregation and cell lethality. This correlation is consistent with the hypothesis that these problems are associated with a highly forked chromosome structure. The detrimental effects of such chromosomes are reduced or eliminated by any environmental or genetic modification that reduces replication overlap. We therefore suggest that the observed morphological alterations of $n r d A 101$ strain at $42^{\circ} \mathrm{C}$ could be ascribed to the activity of an inaccurate replication apparatus. The impaired replication hyperstructure made with a deficient RNR101 protein (Guarino et al., 2007a) stops more frequently than a wild type hyperstructure. In cells with a high degree of replication 

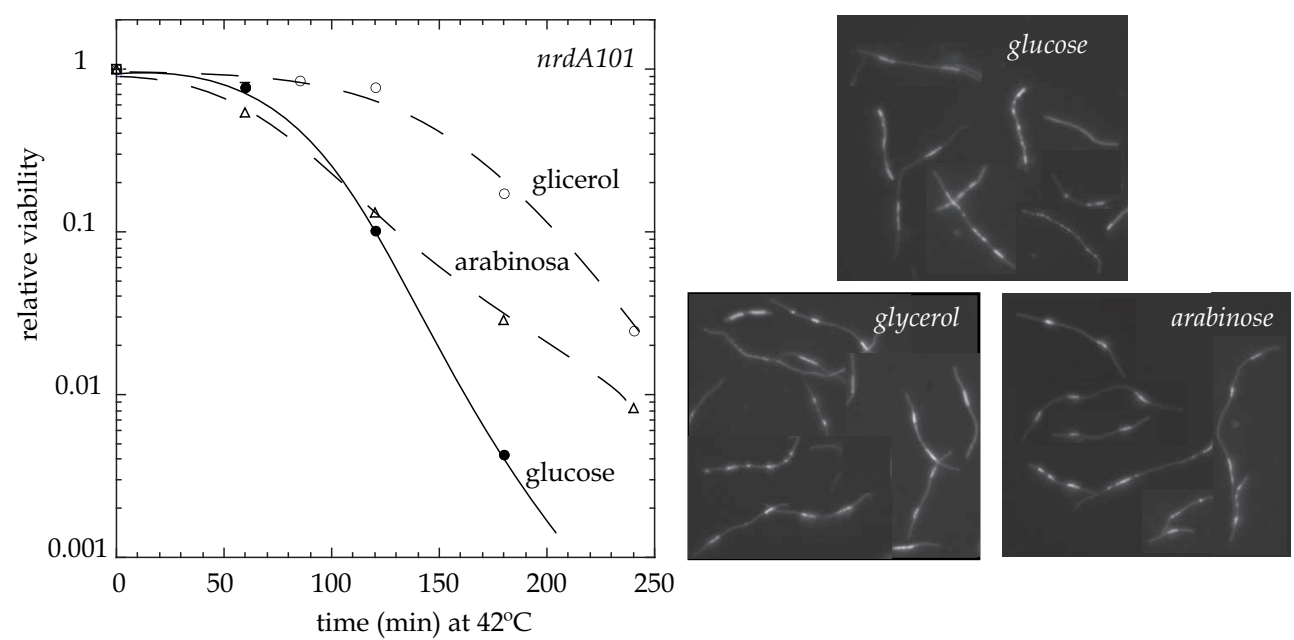

Fig. 5. Cell viability and nucleoid segregation of $n r d A 101$ growing with different carbon sources after the shift to $42^{\circ} \mathrm{C}$.

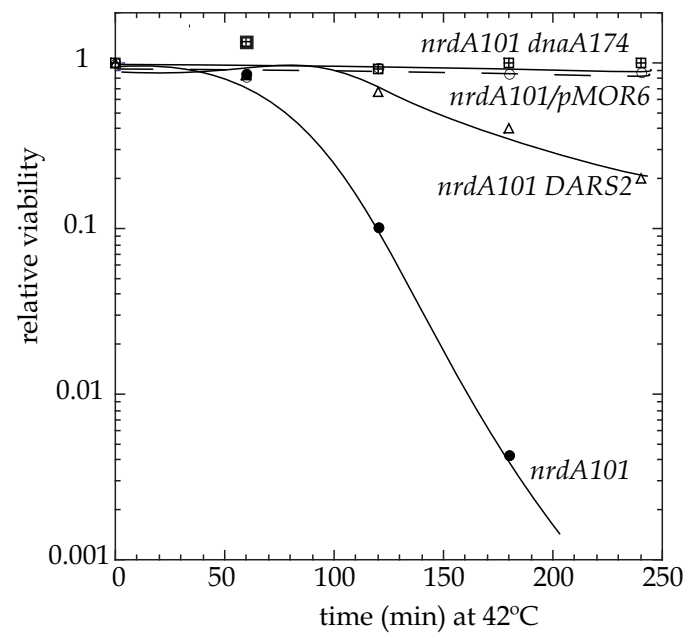

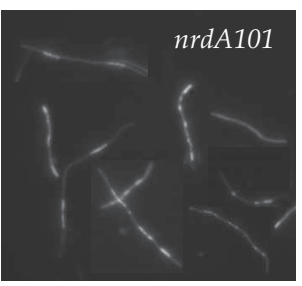
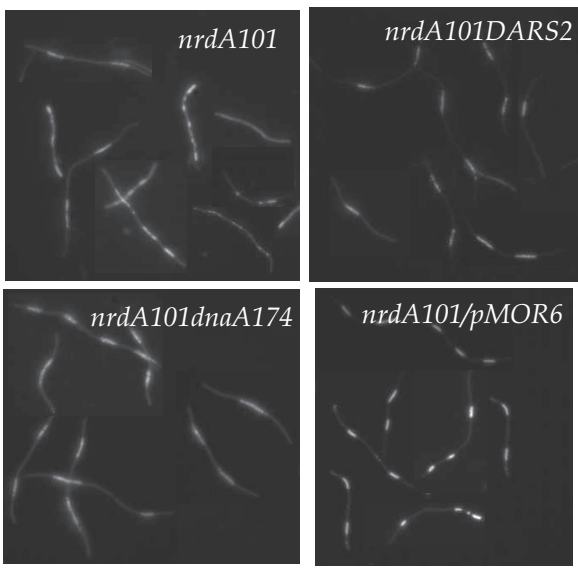

Fig. 6. Cell viability and nucleoid segregation of $n r d A 101$ derivatives after the shift to $42^{\circ} \mathrm{C}$.

overlaps, stalled forks have less opportunity to be repaired and restarted and this interferes with subsequent forks. This results in chromosomal abnormalities, disrupted chromosome and nucleoid segregation, loss of cell division, and, finally, cell death.

DNA topology has been found to play an important role in the segregation of duplicated chromosomes (Dasgupta et al., 2000; Holmes \& Cozarelli, 2000). Consequently, a disturbed DNA topology due to a highly forked chromosome structure, could contribute to the altered nucleoid segregation observed in the $n r d A 101$ mutant at $42^{\circ} \mathrm{C}$. Fork collisions and topological changes would be reduced, or even prevented, in $n r d A 101$ strains at $42^{\circ} \mathrm{C}$ by 
inhibiting new initiations of replication (Salguero et al., 2011), or by diminishing the overlap of replication rounds.

\section{The number of replication rounds in the chromosome limits the replication rate of individual forks}

In the nrdA101 strain growing at the permissive temperature we have found that the number of forks per chromosome was reduced and the elongation rate was increased by the presence of the dnaA174 allele or of extra copies of datA and by the deletion of the DARS2 sequence (Table 2). Reduction of chromosome replication overlaps, with the associated lowering of the ori/ter ratio, together with an increased replication rate, have been also found in strains containing different defective $d n a A$ alleles, such as dnaA204 (Torheim et al., 2001), dnaA46, dnaA174, and dnaA345 (Gon et al., 2006; Morigen et al., 2009), as well as in wild type cells containing extra copies of datA sequence which is believed to reduce the availability of DnaA protein (Morigen et al., 2003). Similar results have also been obtained in studies of $h n s$ (Atlung \& Hansen, 2002) and ihf mutants (von Freiesleben et al., 2000). Additionally, we have also shown by growing the $n r d A 101$ mutant in poor media at $30^{\circ} \mathrm{C}$, that the improvement in the replication fork progression is accompanied by a decrease in replication overlap.The correlation between these effects has been well established but the mechanism remains elusive.

It is difficult to decide whether the reduction in the number of forks is the consequence of an increased replication rate (as ori/ter $=2 \mathrm{n}, \mathrm{n}=\mathrm{C} / \tau$ ), or whether the increase in the replication rate is the consequence of the reduction of the number of forks (ori/ter ratio). The first proposition implies that the activities of DnaA, HNS, and IHF affect the elongation rate directly or indirectly. This is plausible as DnaA protein has 308 binding sites in the bacterial genome (Schaper \& Messer, 1995) and, furthermore, it is a transcriptional regulator controlling the expression of several replication genes (reviewed in Messer \& Weigel, 2003). Therefore, deficiency of DnaA protein, as well as HNS and IHF, might well allow the replication forks to run faster. Moreover, a deficiency of DnaA protein increases $n r d A B$ operon expression (Lobner-Olesen et al., 2008; Gon et al., 2006), which might also increase the velocity of the replication forks (Herrick \& Sclavi, 2008). However, as explained above (2.3.1 this chapter), the over-expression of the $n r d A B$ operon does not necessarily increase the actual supply of the dNTPs used in DNA replication. In addition, the growth of cells in poor carbon source media is not known to affect $n r d A B$ gene expression, and a decrease in replication rate in wild type cells has been observed under poor media conditions (Michelsen et al., 2003). Furthermore, it has been reported that deletion of DnaA box R4 suppresses replication elongation defects in $g y r B$ mutant strains as a consequence of the lowering of initiation frequency (Stepankiw et al., 2009) indicating that no transcriptional factor is required to increase the replication rate. Hence the first proposition, in which faster replication forks are responsible for there being fewer forks, is difficult to justify.

The second proposition is that the elongation rate increased as a consequence of the reduction of the number of forks or the replication overlap. This reduction in the number of the forks would be caused by the deficiency of any factor required for the initiation step since this would result in the delay of the initiation of replication.

In the above work, we have shown that a decrease in the growth rate of the nrdA101 mutant, due to growth on poor carbon sources, improves the elongation rate of chromosome replication, which is the same as to say that $C$ decreases when $\tau$ increases. From the 
algorithm $C=n \tau$ one can conclude that any increase in the cell doubling time, should lead to a decrease in the $n$ value, or the number of forks per chromosome. Nevertheless, we have shown that increasing $\tau$ also decreases the $C$ value. Even though elongation rate in wild type strains is expected to be lower under decreased growth rate (Michelsen et al., 2003) we can infer that a reduction in the number of forks per chromosome in the $n r d A 101$ strain with a extremely slow replication rate could also be a cause of improvement of the replication rates.

An unified explanation for all the results presented here is difficult to find. Clearly though, the underlying mechanism should explain the precise correlation between initiation and elongation that tunes DNA replication to any environmental circumstance. Whatever the nature of this mechanism, reduction in the number of forks per chromosome or decreased overlapping of consecutive replication rounds might increase the elongation rate by providing $i$ ) a better overall chromosome structure, including discrete regional organization and supercoiling domains, ii) an increased availability of a limiting constituent required for replication and/or for segregation, and iii) an increased time for the repair and restart of a stalled fork so as to avoid collision with the next fork. This homeostatic regulation between the numbers and velocities of forks would also explain how the replication rate compensates for widely varying replication origins and activities in eukaryotes (Conti et al., 2007).

\section{Balance between the number of origins and elongation rates as a general regulatory mechanism in the control of eukaryotic cell cycle}

In eukaryotic cells, the DNA replication program is organized according to multiple tandem replicons that span each chromosome. Each replicon is replicated bidirectionally by a pair of replication forks that increase their rates up to three fold towards the end of $S$ phase. Furthermore, the rate of the replication fork progression varies up to ten-fold or more depending on the distance between origins in different conditions or cell types (Housman \& Huberman, 1975; reviewed in Herrick, 2010). Two replication regimes with distinct kinetics govern duplication of the genome: in the first half of the $S$ phase, when the gene-rich euchromatin is predominantly replicated, the density of the activated replication origins steadily increases to about twice the initial value; during the second half of the $S$ phase, when the gene-poor heterochromatin tends to be replicated, the density of active replication origins increases substantially by about ten fold (Herrick \& Bensimon, 2008). It has been proposed that this mechanism would guarantee the rapid and complete duplication of the genome. Nevertheless, in mammalian cells the relationship between origin activation, the size of replicons $(50-300 \mathrm{~kb})$ and the existence of multiple potential origins remains to be elucidated (Herrick, 2010).

The efficient duplication of the eukaryotic genome depends on the orderly activation of the origins, estimated to be ten thousand, and on the proper progression of their forks. The coordinated activation of origins is insufficient on its own to account for timely completion of genome duplication when interorigin distances vary significantly and fork velocities are constant. Therefore the coordination and compensation between origin spacing and fork progression may be one of the mechanisms for the complete duplication of the genome in the limited amount of time of the $S$ phase. By using a single-molecule approach based on molecular combing, the interorigin distances and replication fork velocities over extensive regions of the genome have been measured in both primary keratinocytes and cancer cells (Conti et al., 2007). This study provides evidence for the direct correlation between the interorigin distances and the replication rates, insofar as the further the origins are from one 
another, the faster the forks progress. These results are in agreement with the results of this and other studies of $E$. coli, which show a correlation between the frequency of initiation (ori/ter ratio) and the replication fork rates.

Figure 3 in Conti et al., 2007 shows a significant linear correlation between these two parameters in eukaryotic cells, consistent with a biological mechanism that coordinates replication fork progression with interorigin distance. The mechanism that allows replication forks to adjust their speed is unknown. Nevertheless the possibilities for the nature of this mechanism are similar to the ones proposed above for E. coli. A feedback mechanism might be based on the accumulation of torsional strain as incoming fork approach each other and the length of DNA to be replicated decreases. However, a mechanism based only on mechanical stress would strongly limit the possibility of modification and adaptation of the fork rates. The concentration of dNTP could also play a role in regulating fork velocity (Anglana et al., 2003). Supporting this notion, it has been shown that the kinase Chk1 plays an essential role in $S$ phase progression through regulation of RNR2 expression (Naruyama et al., 2008), although ectopic expression of RNR2 failed to rescue the S phase arrest observed in Chk1-depleted cells, suggesting the presence of Chk1 target(s) for completion of $S$ phase in addition to or other that RNR2. The observation of dynamically regulated adjacent forks also supports the idea that dNTP pool sizes alone are not implicated in the observed changes in fork velocity (Conti et al., 2007). Additionally, intracellular dNTP pool sizes are expected to increase (as replication rate increase), rather than decrease, during $S$ phase (Malinsky et al., 2001). Therefore, although the size of the dNTP pool could be globally responsible for fork velocity, it would not be responsible for the local control and dynamic correlation between adjacent forks; this must involve other factors, for example, the processivity of DNA helicases and toposiomerases (Conti et al., 2007).

\section{Concluding remarks}

In this work we show that reducing the number of replication forks per chromosome in E. coli improves the amount of DNA that a thermosensitive $n r d A$ mutant strain is able to synthesize at restrictive conditions. Activity of the RNR101 at $42^{\circ} \mathrm{C}$ has been proposed to be maintained due to the protection of the thermolabile protein by the replication hyperstructure; therefore, the effect we have found may be related to the processivity of the replication hyperstructure. More specifically, in our hypothesis, the processivity of the replication hyperstructure is improved by the lowering of the number of the replication forks along the chromosome, i.e. by reducing the overlap. Such a relationship between processivity and the number of replication forks could be explained by 1) variations in the availability of some limiting hyperstructure component which might lead to assembly of an inefficient hyperstructure when a high number of forks compete for this component, or 2) the structural constraints caused by a chromosome undergoing several rounds of replication running at the same time. Results from other research groups, reviewed above, and comparison with DNA replication in eukaryotes provide further evidence that, in widely different systems, the initiation and the elongation of chromosome replication are not independent processes.

\section{Acknowledgements}

We are very grateful to Kirsten Skarstad and Tsutomu Katayama for bacterial strains and plasmids. We especially thank Encarna Ferrera for her technical help. This work was 
supported by grant BFU2007-63942 from the Ministerio de Ciencia e Innovación. IS, CM and MAS-R acknowledge the studentship from Junta de Extremadura.

\section{References}

Anglana, M., Apiou, F., Bensimon, A. \& Debatisse, M. (2003). Dynamics of DNA replication in mammalian somatic cells: nucleotide pool modulates origin choice and interorigin spacing. Cell 114: 385-94.

Atlung, T. \& Hansen, F.G. (2002). Effect of different concentrations of H-NS protein on chromosome replication and the cell cycle in Escherichia coli. J Bacteriol 184: 18431850.

Blinkova, A., Hermandson, M. \& Walker J. R. (2003). Suppression of Temperature-Sensitive Chromosome Replication of an Escherichia coli dnaX(Ts) Mutant by Reduction of Initiation Efficiency. J Bacteriol. 185: 3583-3595.

Bierne, H. \& Michel, B. (1994). When replication forks stop. Mol Microbiol. 13: 17-23.

Bussiere, D.E. \& Bastia, D. (1999). Termination of DNA replication of bacterial and plasmid chromosomes. Mol Microbiol. 31: 1611-1618.

Conti, C., Sacca, B., Herrick, J., Lalou, C., Pommier, Y. \& Bensimon, A. (2007). Replication fork velocities at adjacent replication origins are coordinately modified during DNA replication in human cells. Molecular Biology of the Cell. 18: 3059-3067.

Cooper, S. \& Helmstetter, C.E. (1968). Chromosome replication and the division cycle of Escherichia coli B/r. J Mol Biol 31: 519-540.

Dasgupta, S., Maisnier-Patin, S. \& Nordstrom, K. (2000). New genes with old modus operandi. The connection between supercoiling and partitioning of DNA in Escherichia coli. EMBO Rep 1: 323-327.

Dix, D. E. \& Helmstetter, C. E. (1973). Coupling between chromosome completion and cell division in Escherichia coli. J Bacteriol 115: 786-95.

Fuchs, J. A., Karlstrom, H. O., Warner, H. R. \& Reichard, P. (1972). Defective gene product in dnaF mutant of Escherichia coli. Nat New Biol 238: 69-71.

Fujimitsu K, Senriuchi T, Katayama, T. (2009). Specific genomic sequences of E. coli promote replicational initiation by directly reactivating ADP-DnaA. Genes Dev. 23: 1221-33.

Gines-Candelaria, E., Blinkova, A. \& Walker, J. R. (1995). Mutations in Escherichia coli dnaA which suppress a dnaX(Ts) polymerization mutation and are dominant when located in the chromosomal allele and recessive on plasmids. J Bacteriol 177: 705715.

Gon, S., Camara, J.E., Klungsoyr, H.K., Crooke, E., Skarstad K. \& Beckwith, A.J. (2006). A novel regulatory mechanism couples deoxyribonucleotide synthesis and DNA replication in Escherichia coli. EMBO J 25: 1137-1147.

Grompe, M., Versalovic, J., Koeuth, T. \& Lupski, J.R. (1991). Mutations in the Escherichia coli dnaG gene suggest coupling between DNA replication and chromosome partitioning. J Bacteriol. 173: 1268-1278.

Guarino, E., Jiménez-Sánchez, A. \& Guzmán, E. C. (2007). Defective ribonucleoside diphosphate reductase impairs replication fork progression in Escherichia coli. J Bacteriol 189: 3496-501.

Guarino, E., Salguero, I., Jiménez-Sánchez, A. \& Guzmán, E. C. (2007). Double-strand break generation under deoxyribonucleotide starvation in Escherichia coli. J Bacteriol 189: $5782-6$. 
Guzmán, E. C., Caballero, J. L. \& Jiménez-Sánchez, A. (2002). Ribonucleoside diphosphate reductase is a component of the replication hyperstructure in Escherichia coli. Mol Microbiol 43: 487-95.

Guzmán, E. C., Guarino, E., Riola, J. \& Jiménez-Sánchez, A. (2003). Ribonucleoside diphosphate reductase is a functional and structural component of the replication hyperstructure in Escherichia coli. Rec Res Devel Mol Biol 1: 29-43.

Hanke, P. D. \& Fuchs, J. A. (1983). Regulation of ribonucleoside diphosphate reductase mRNA synthesis in Escherichia coli. J Bacteriol 154: 1040-1045.

Herrick, J. (2010) The dynamic replicon: adapting to a changing cellular environment. Bioessays 32:153-64.

Herrick, J. \& Sclavi, B. (2007). Ribonucleotide reductase and the regulation of DNA replication: an old story and an ancient heritage. Mol Microbiol 63: 22-34.

Herrick, J. \& Bensimon, A. (2008). Global regulation of genome duplication in eukaryotes: an overview from the epifluorescence microscope. Chromosoma 117: 243-260.

Hill, T.M, Sharma, B., Valjavec-Gratian, M. \& Smith, J. (1997). sfi-independent filamentation in Escherichia coli is lexA dependent and requires DNA damage for induction. J Bacteriol 179 :1931-9.

Holmes, V.F. \& Cozzarelli, N.R. (2000). Closing the ring: links between SMC proteins and chromosome partitioning, condensation, and supercoiling. Proc Natl Acad Sci USA 97: 1322-1324.

Housman, D., \& Huberman, J.A. (1975). Changes in the rate of DNA replication fork movement during S phase in mammalian cells.J Mol Biol 94: 173-181.

Jaffe, A., D'Ari, R. \& Norris, V. (1986). SOS-independent coupling between DNA replication and cell division in E. coli. J. Bacteriol.165, 66-71.

Jannière, L., Canceill, D, Suski, C., Kanga, S., Dalmais B, Lestini, R., Monnier, A.F., Chapuis, J., Bolotin, A., Titok, M., Le Chatelier, E. \& Ehrlich, S.D. Genetic evidence for a link between glycolysis and DNA replication. (2007). PLoS One 2(5):e447.

Jiménez-Sánchez, A. \& Guzmán, E.C. (1988). Direct procedure for the determination of the number of replication forks and the reinitiation fraction in bacteria. Comput Appl Biosci 4: 431-433.

Kawakami, H., Iwura, T., Tanaka, M. \& Sekimizu, T. (2001). Arrest of cell division and nucleoid partition by genetic alterations in the sliding clamp of the replicase and in DnaA. Mol. Genet. Genomics 266: 167-79.

Kaguni, J. M. (2006). DnaA: Controlling the Initiation of Bacterial DNA Replication and More. Annu. Rev. Microbiol 60: 351-71.

Kim, J., Wheeler, L.J., Shen, R., \& Mathews, C.K. (2005). Protein-DNA interactions in the T4 dNTP synthetase complex dependent on gene 32 single-stranded DNA-binding protein. Mol Microbiol 55: 1502-1514.

Kuzminov, A. (1995). Collapse and repair of replication forks in Escherichia coli. Mol Microbiol 16: 373-384.

Kitagawa, R., Mitsuki, H, Okazaki T. \& Ogawa, T. (1996). A novel DnaA protein-binding site at $94.7 \mathrm{~min}$ on the Escherichia coli chromosome. Mol Microbiol 19:1137-47.

Kitagawa, R., Ozaki, T., Moriya, S. \& Ogawa, T. (1998). Negative control of replication initiation by a novel chromosomal locus exhibiting exceptional affinity for Escherichia coli DnaA protein. Genes Dev 12 :3032-43. 
Kurokawa, K., Nishida, S., Emoto, A., Sekimizu, K., \& Katayama, T. (1999). Replication cycle-coordinated change of the adenine nucleotide-bound forms of DnaA protein in Escherichia coli. Embo J 18: 6642-6652.

Løbner-Olesen, A., Slominska-Wojewodzka, M., Hansen, F. G. \& Marinus, M. G. (2008). DnaC Inactivation in Expression of Nucleotide Biosynthesis Genes. PLoS ONE 3(8): e2984.

Malínsky, J., Koberna, K., Stanĕk, D., Masata, M., Votruba, I. \& Raska, I. (2001). The supply of exogenous deoxyribonucleotides accelerates the speed of the replication fork in early S-phase. J Cell Sci. 114 :747-50.

Maisnier-Patin, S., Nordstrom, K., y Dasgupta, S. (2001). Replication arrests during a single round of replication of the Escherichia coli chromosome in the absence of DnaC activity. Mol Microbiol 42: 1371-1382.

Mathews, C. K. (1993). Enzyme organization in DNA precursor biosynthesis. Prog Nuc Ac Res 44: 167-203.

Messer, W. \& Weigel, C. (2003). DnaA as a transcription regulator. Methods Enzymol. 370: 338-49.

Michel, B., Grompone, G., Flores, M.J., \& Bidnenko, V. (2004). Multiple pathways process stalled replication forks. Proc Natl Acad Sci U S A 101: 12783-12788.

Michelsen, O., Teixeira de Mattos, M.J, Jensen, P.R. \& Hansen, F.G. (2003). Precise determinations of C and D periods by flow cytometry in Escherichia coli K-12 and B/r. Microbiology 149: 1001-10.

Molina, F. \& Skarstad, K. (2004). Replication fork and SeqA focus distributions in Escherichia coli suggest a replication hyperstructure dependent on nucleotide metabolism. Mol Microbiol 52: 1597-612.

Morigen, Boye, E., Skarstad, K. \& Løbner-Olesen A. (2001). Regulation of chromosomal replication by DnaA protein availability in Escherichia coli: effects of the datA region. Biochem. et Bioph. Acta 1521: 73-80.

Morigen, Løbner-Olesen, A. \& Skarstad K. (2003). Titration of the Escherichia coli DnaA protein to excess datA sites causes destabilization of replication forks, delayed replication initiation and delayed cell division. Mol Microbiol 50: 349-62.

Morigen, Odsbu, I., Skarstad, K. (2009). Growth rate dependent numbers of SeqA structures organize the multiple replication forks in rapidly growing Escherichia coli. Genes Cells 14: 643-657.

Naruyama H, Shimada M, Niida H, Zineldeen DH, Hashimoto Y, Kohri K, Nakanishi M (2008). Essential role of Chk1 in S phase progression through regulation of RNR2 expression. Biochem Biophys Res Commun. 374: 79-83.

Nordlund, P. \& Reichard, P. (2006). Ribonucleotide reductases. Annu. Rev. Biochem 75: 681706.

Odsbu, I., Morigen \& Skarstad, K. (2009). A reduction in ribonucleotide reductase activity slows down the chromosome replication fork but does not change its localization. PLoS One 4 (10), e7617.

Olliver, A., Saggioro, C., Herrick, J. \& Sclavi, B. (2010). DnaA-ATP acts as a molecular switch to control levels of ribonucleotide reductase expression in Escherichia coli. Mol Microbiol 76: 1555-71. 
Pato, M. L. (1979). Alterations of deoxyribonucleoside triphosphate pools in Escherichia coli: effects on deoxyribonucleic acid replication and evidence for compartmentation. $J$ Bacteriol 140: 518-24.

Pritchard, R.H. \& Zaritsky, A. (1970). Effect of Thymine Concentration on the replication velocity of DNA in a thymineless mutant of Escherichia coli. Nature 226: 126-130.

Riola, J., Guarino, E., Guzmán, E. C. \& Jiménez-Sánchez, A. (2007). Differences in the degree of inhibition of NDP reductase by chemical inactivation and by the thermosensitive mutation nrdA101 in Escherichia coli suggest an effect on chromosome segregation. Cell Mol Biol Lett 12: 70-81.

Salguero, I, López Acedo, E. \& Guzmán, E. C. (2011). Overlap of replication rounds disturbs the progression of replicating forks in a ribonucleotide reductase mutant of Escherichia coli. Microbiology 157: 1955-1967.

Sánchez Romero, M.A., Molina, F. \& Jiménez-Sánchez, A. (2010). Correlation between ribonucleoside-diphosphate reductase and three replication proteins in Escherichia coli. BMC Molecular Biology doi: 10.1186/1471-2199-11-11.

Sekimizu, K., Bramhill, D. \& Kornberg A. (1987). ATP activates DnaA protein in initiating replication of plasmids bearing the origin of the E. coli chromosome. Cell 50: 259-65.

Schaper, S. \& Messer, W. (1995). Interaction of the initiator protein DnaA of Escherichia coli with its DNA target. J Biol Chem. 270: 17622-6.

Skarstad, K., Steen, H. B. \& Boye, E. (1985). Escherichia coli DNA distributions measured by flow cytometry and compared with theoretical computer simulations. J Bacteriol 163: 661-8.

Skovgaard, O. \& Lobner-Olesen, A. (2005). Reduced initiation frequency from oriC restores viability of a temperature-sensitive Escherichia coli replisome mutant. Microbiology 151: 963-73.

Stepankiw, N., Kaidow, A., Boye, E. \& Bates, D. (2009). The right half of the Escherichia coli replication origin is not essential for viability, but facilitates multi-forked replication. Mol Microbiol. 74: 467-479.

Sueoka, N. \& Yoshikawa, H. (1965). The chromosome of Bacillus subtilis. I. Theory of marker frequency analysis. Genetics 52: 747-757.

Torheim, N.K, Boye , E., Løbner-Olesen, A., Stokke, T. \& Skarstad, K. (2000). The Escherichia coli SeqA protein destabilizes mutant DnaA204 protein. Mol Microbiol. 37: 629-38.

Uhlin, U. \& Eklund, H. (1994). Structure of ribonucleotide reductase protein R1. Nature 13: 533-539.

Von Freiesleben, U., Rasmussen, K.V., Atlung, T., \& Hansen, F.G. (2000). Rifampicinresistant initiation of chromosome replication from oriC in ihf mutants. Mol Microbiol 37: 1087-1093.

Werner, R. (1971). Nature of DNA precursors. Nature New Biol 233: 99-103.

Wheeler, L.J., Rajagopal, I. \& Mathews, C. K. (2005). Stimulation of mutagenesis by proportional deoxyribonucleoside triphosphate accumulation in Escherichia coli. DNA repair 4, 1450-1456.

Zaritsky, A. \& Pritchard, R.H. (1971). Replication time of the chromosome in thymineless mutants of Escherichia coli. J Mol Biol 60: 65-74.

Zaritsky, A., N. Vischer \& Rabinovitch, A. (2007). Changes of initiation mass and cell dimensions by the 'eclipse'. Mol Microbiol 63: 15-21. 


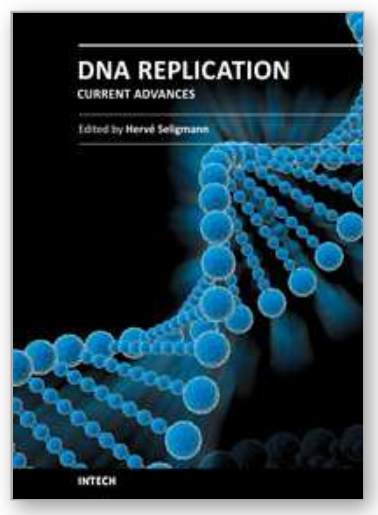

\section{DNA Replication-Current Advances}

Edited by Dr Herve Seligmann

ISBN 978-953-307-593-8

Hard cover, 694 pages

Publisher InTech

Published online 01, August, 2011

Published in print edition August, 2011

The study of DNA advanced human knowledge in a way comparable to the major theories in physics, surpassed only by discoveries such as fire or the number zero. However, it also created conceptual shortcuts, beliefs and misunderstandings that obscure the natural phenomena, hindering its better understanding. The deep conviction that no human knowledge is perfect, but only perfectible, should function as a fair safeguard against scientific dogmatism and enable open discussion. With this aim, this book will offer to its readers 30 chapters on current trends in the field of DNA replication. As several contributions in this book show, the study of DNA will continue for a while to be a leading front of scientific activities.

\section{How to reference}

In order to correctly reference this scholarly work, feel free to copy and paste the following:

Elena C. Guzmán, Israel Salguero, Carmen Mata Martin, Elena López Acedo, Estrella Guarino, Ma Antonia Sánchez-Romero, Vic Norris and Alfonso Jiménez-Sánchez (2011). Relationship between Fork Progression and Initiation of Chromosome Replication in E. coli, DNA Replication-Current Advances, Dr Herve Seligmann (Ed.), ISBN: 978-953-307-593-8, InTech, Available from: http://www.intechopen.com/books/dna-replicationcurrent-advances/relationship-between-fork-progression-and-initiation-of-chromosome-replication-in-e-coli

\section{INTECH}

open science | open minds

\section{InTech Europe}

University Campus STeP Ri

Slavka Krautzeka 83/A

51000 Rijeka, Croatia

Phone: +385 (51) 770447

Fax: +385 (51) 686166

www.intechopen.com

\section{InTech China}

Unit 405, Office Block, Hotel Equatorial Shanghai

No.65, Yan An Road (West), Shanghai, 200040, China

中国上海市延安西路65号上海国际贵都大饭店办公楼 405 单元

Phone: +86-21-62489820

Fax: +86-21-62489821 
(C) 2011 The Author(s). Licensee IntechOpen. This chapter is distributed under the terms of the Creative Commons Attribution-NonCommercialShareAlike-3.0 License, which permits use, distribution and reproduction for non-commercial purposes, provided the original is properly cited and derivative works building on this content are distributed under the same license. 